\title{
The correlation between kidney volume and measured glomerular filtration rate in an Asian ADPKD population: a prospective cohort study
}

Bunyong Phakdeekitcharoen ${ }^{1 *}$, Watcharapong Treesinchai ${ }^{1}$, Pornphan Wibulpolprasert ${ }^{2}$, Sarinya Boongird ${ }^{1}$ and Pinkael Klytrayong ${ }^{1}$

\begin{abstract}
Background: Autosomal dominant polycystic kidney disease (ADPKD) is the most common hereditary kidney disorder that leads to end stage renal disease (ESRD). Cyst expansion in ADPKD is strongly associated with the decline in renal function. However, the correlation between total kidney volume (TKV) and glomerular filtration rate (GFR) at an early stage has not been well demonstrated. There is growing evidence that utilization of estimated GFR (eGFR) may induce misleading information in a population with near normal renal function. Therefore, a more accurate method is essential.

Methods: A prospective cohort of ADPKD patients was conducted with clinical data and laboratory collection. Measured GFR (mGFR) was assessed by iohexol plasma clearance method using ultra performance liquid chromatography. eGFR was calculated using the CKD-EPI equation. Kidney volumes were evaluated using MRI imaging protocol.

Results: Thirty two patients completed the study. The mean age was 56 years old. The mean initial mGFR was $83.8 \mathrm{~mL} /$ $\mathrm{min} / 1.73 \mathrm{~m}^{2}$. The mean change in $\mathrm{mGFR}$ per year was $-2.99 \mathrm{~mL} / \mathrm{min} / 1.73 \mathrm{~m}^{2} /$ year. The mean initial height-adjusted TKV (htTKV) was $681.0 \mathrm{~mL} / \mathrm{m}$. The mean percentage change in htTKV per year (\% $\mathrm{htTKV} / \mathrm{y})$ was $4.77 \% /$ year. mGFR had a better association with clinical parameters than eGFR. Initial mGFR was significantly and inversely correlated with initial $\mathrm{htTKV}$ and age. The percentage change in mGFR per year was significantly and inversely correlated with the \% $\mathrm{htTKV} / \mathrm{y}$ and 24-hr urine albumin. The \% $\mathrm{\Delta htTKV/y}$ was significantly correlated with initial htTKV.

Conclusions: Our studies demonstrated that mGFR using iohexol is a more reliable and accurate method than eGFR for evaluating GFR changes in the early stages of ADPKD patients. There is a strong inverse correlation between kidney volume and mGFR in an Asian ADPKD population. The initial htTKV is a good predictor of kidney volume progression. The $\% \Delta \mathrm{htTKV} / \mathrm{y}$ is a good early surrogate marker for the decline in renal function. 24-hr urine albumin is also a good indicator for renal progression.
\end{abstract}

Keywords: Autosomal dominant polycystic kidney disease (ADPKD), Measured GFR (mGFR), lohexol, Total kidney volume (TKV), Ultra performance liquid chromatography (UPLC)

\footnotetext{
* Correspondence: bunyong.pha@mahidol.ac.th

'Division of Nephrology, Department of Medicine, Faculty of Medicine, Ramathibodi Hospital, Mahidol University, 10400 Bangkok, Thailand

Full list of author information is available at the end of the article
}

C C The Author(s). 2021 Open Access This article is licensed under a Creative Commons Attribution 4.0 International License, which permits use, sharing, adaptation, distribution and reproduction in any medium or format, as long as you give appropriate credit to the original author(s) and the source, provide a link to the Creative Commons licence, and indicate if changes were made. The images or other third party material in this article are included in the article's Creative Commons licence, unless indicated otherwise in a credit line to the material. If material is not included in the article's Creative Commons licence and your intended use is not permitted by statutory regulation or exceeds the permitted use, you will need to obtain permission directly from the copyright holder. To view a copy of this licence, visit http://creativecommons.org/licenses/by/4.0/ The Creative Commons Public Domain Dedication waiver (http://creativecommons.org/publicdomain/zero/1.0/) applies to the data made available in this article, unless otherwise stated in a credit line to the data. 


\section{Background}

Autosomal dominant polycystic kidney disease (ADPKD) is the most common hereditary kidney disorder that leads to end stage renal disease (ESRD) [1, 2]. Clinical presentation varies widely, including hypertension, hematuria, proteinuria and renal insufficiency [3]. It is characterized by gradual renal enlargement and cyst growth prior to loss of renal function [4]. The average age of entry into ESRD is 57 years [5]. The kidney function remains stable in ADPKD patients for years, followed by a sharp decline in glomerular filtration rate (GFR) once a critical renal size is reached $[6,7]$. Clinical parameters associated with decline in kidney function in ADPKD patients have been gradually reported [8-10]. However, the lack of a sensitive measure of disease progression in the early stages of ADPKD has caused problems in the development of prevention and of therapeutic agents [11-13].

Imaging methods that reliably and accurately measure total kidney volume (TKV) in ADPKD patients have been developed utilizing magnetic resonance imaging (MRI) and computerized tomography [14-16]. The Consortium of Radiologic Imaging Studies of Polycystic Kidney Disease (CRISP) study has demonstrated that the expansion of cysts in patients with ADPKD is strongly associated with the decline in renal function [14]. Higher rates of kidney enlargement are associated with a more rapid decline in renal function. However, the correlation between TKV and the accuracy of GFR changes at an early stage has not been well demonstrated. The results of clinical trials have shown no beneficial effects of sirolimus or everolimus therapy against progressive GFR decline in two large cohorts of ADPKD patients using estimated GFR by calculation, namely the Chronic Kidney Disease Epidemiology Collaboration (CKD-EPI) and the Modification of Diet in Renal Disease (MDRD) equations, that is based on serum creatinine levels represented as a marker of glomerular filtration [12, 13]. These formulas have been constantly evaluated and there is growing evidence that their utilization may induce misleading information, especially in population with normal or near normal renal function [17-21]. The authors in previous studies have suggested that in early stages of ADPKD, the variability in serum creatinine levels may be explained by creatinine production related to muscle mass or protein intake rather than glomerular filtration [22]. Therefore, direct measurements of GFR by gold-standard techniques depended on the use of exogenous markers such as inulin, iohexol or radio-labeled tracers, which would be essential in order to assess the accuracy of GFR decline in the studied population [23-25].

In this study, we compared the efficacy between eGFR using CKD-EPI and mGFR using iohexol plasma clearance for evaluating GFR changes in the early stages of patients with ADPKD and investigated the relationship between clinical parameters, total kidney volume and renal function and identified the risk factors associated with the progression in kidney structure and function decline in the Thai ADPKD population.

\section{Methods \\ Study population}

A prospective cohort study was conducted, with test subjects being patients under the care of the nephrology division, Department of Medicine, Ramathibodi Hospital, during 2015-2019. The inclusion criteria are as follows: diagnosis of ADPKD (by family history, the Ravine's criteria [26], and genetic analysis [27]), age more than 18 years, clinically stable, eGFR $\geq 30 \mathrm{~mL} / \mathrm{min} / 1.73 \mathrm{~m}^{2}$ by CKD-EPI and willing to join the study. The exclusion criteria included undergoing transplantation, contraindication for MRI, pregnancy, and a high mortality disease such as cancers. At each visit, the medical history, adverse events, medical changes, and hospitalizations were recorded. Other clinical data were prospectively collected including: age of enrollment, weigh, height, body mass index (BMI), mean systolic blood pressure (SBP), mean diastolic blood pressure (DBP), serum creatinine $(\mathrm{sCr})$, serum uric, fasting blood sugar, and LDL-cholesterol. 24-h urine protein and 24-h urine albumin were collected after being enrolled in the study for 4 weeks. Kidney volume and GFR were measured annually. All methods were performed in accordance with the relevant guidelines and regulations of the institute. Written informed consent was obtained from all patients. The study was approved by the Institutional Ethics Committee on Human Rights Related to Research Involving Human Subjects of the Ramathibodi Hospital, Mahidol University.

\section{GFR measurements}

mGFR was determined by the plasma clearance of iohexol. Briefly, iohexol was administered as Omnipaque $300 \mathrm{mg}$ I/mL (Amersham Health, South Plainfield, NJ, USA). Venous blood samples were collected at four time points $(120,180,240$, and $300 \mathrm{~min})$ after a single 5-ml intravenous injection of iohexol. Plasma concentration of iohexol was determined by ultra performance liquid chromatography (UPLC). The UPLC analytical system was performed with Agilent technologies (Agilent Technologies, Wilmington, DE, USA). The system was connected to the autosampler UV detector. The manager software was ChemStation. The intra-assay and inter-assay variant of UPLC were 0.94 and $2.44 \%$, respectively. A curve was obtained expressing iohexol elimination. We calculated measured GFR by using the one-compartment model, often referring as the slope-intercept technique. The onecompartment clearance, $\mathrm{Cl}_{1}$, is the ratio between the injected amount of iohexol dose and the area under the 
slow-compartment (final monoexponential part) of plasma concentration curve. Since the $\mathrm{Cl}_{1}$ did not include the whole area of plasma concentration curve, the mathematical correction for the first fast exponential curve is needed. Herein, we used the correction equation proposed by Brochner-Mortensen (BM) to calculate the measured GFR [28]. GFR from BM correction $(\mathrm{mL} / \mathrm{min})=$ $\left(0.990778 \times C l_{1}\right)-\left(0.001218 \times C l_{1}^{2}\right)$. All mGFR results were adjusted for body surface area.

The morning of each iohexol clearance study, serum creatinine concentration was measured with enzymatic method using an automatic device (Architec c16000, Abbott core laboratory, Illinois, USA). eGFR was calculated using the CKD-EPI equation [29].

\section{MRI imaging protocol}

All renal MRI imaging were performed on a 3.0-T MRI scanner (Ingenia MR system; Philips, Best, Netherland). The MRI protocol included sshT2W, IDEAL, non-contrast enhanced T1W, and dynamic contrast enhanced MRI sequences. Following this acquisition of pre-gadolinium images, $0.1 \mathrm{mmol} / \mathrm{kg}$ gadobutrol (Gadovist; $1.0 \mathrm{mmol} / \mathrm{mL}$ ) was injected at $1 \mathrm{~mL} / \mathrm{s}$. The post-gadolinium dynamic imaging was acquired in three initial phase $(25,40$, and $60 \mathrm{~s}$ after contrast administration), and then after a delay of 3,5 , and $10 \mathrm{~min}$, with a bolus tracking technique and fat saturation. Kidney volumes were measured by manually tracing the kidney contours using volume analysis solfware implemented on an Advantage Windows Workstation (4.4, GE Healthcare, Buc, France) as previously prescribed in the study protocol [30]. The sshT2W images were used by the experience radiologists for measuring kidney volumes since this non gadolinium MRI sequence provided reliable kidney volume measurement [30].

\section{Data and Statistical Analysis}

The study was designed as a prospective cohort study. Statistics were analyzed with SPSS V 18.0. Numerical data were shown with mean $\pm S D$ or median (range). Pearson's correlation coefficients were used to examine the association among eGFR or mGFR, the percentage change in eGFR or mGFR per year $(\% \Delta \mathrm{eGFR} / \mathrm{y}$ or $\% \Delta \mathrm{mGFR} / \mathrm{y})$, the percentage change in height-adjusted total kidney volume per year $(\% \Delta \mathrm{htTKV} / \mathrm{y})$, and selected clinical variables. Univariate and multivariate regression analyses were used to determine independent predictors of baseline eGFR or mGFR, $\% \Delta \mathrm{eGFR} / \mathrm{y}$ or $\% \Delta \mathrm{mGFR} / \mathrm{y}$, and $\% \Delta$ htTKV/y. Two-tail $p$ values of $<0.05$ were considered to indicate a statistically significant difference.

\section{Results}

Patients' characteristics

Initially, there were 41 ADPKD patients included in the study. One patient was excluded from the study due to nephrotic syndrome and eight were dropped off due to incomplete follow up. Thirty two ( 25 female and 7 male) patients completed the study. The mean duration of follow-up time was $1.79 \pm 0.58$ years. Table 1 shows the

Table 1 Baseline characteristics and contemporaneous measurements of the patients in this study

\begin{tabular}{|c|c|c|}
\hline & $\begin{array}{l}\text { Complete } \\
\text { study }(N=32)\end{array}$ & $\begin{array}{l}\text { Incomplete } \\
\text { study }(N=8)\end{array}$ \\
\hline Age (years) & $56.3 \pm 12.7$ & $55.3 \pm 11.8$ \\
\hline Female $(\mathrm{N}, \%)$ & $25(78.1 \%)$ & $5(62.5 \%)$ \\
\hline $\mathrm{BMI}\left(\mathrm{Kg} / \mathrm{m}^{2}\right)$ & $23.7 \pm 3.2$ & $23.8 \pm 2.9$ \\
\hline $\mathrm{BMI}>25(\mathrm{~N}, \%)$ & $10(31 \%)$ & $3(37.5 \%)$ \\
\hline $\mathrm{SBP}(\mathrm{mmHg})$ & $130.6 \pm 15.5$ & $130.9 \pm 15.6$ \\
\hline $\mathrm{DBP}(\mathrm{mmHg})$ & $79.2 \pm 8.7$ & $79.5 \pm 8.8$ \\
\hline History of smoking (N, \%) & $5(15.6 \%)$ & $1(12.5 \%)$ \\
\hline Hypertension (N, \%) & $24(75 \%)$ & $6(75 \%)$ \\
\hline Hypertension before 35 year old & $2(6.3 \%)$ & $1(12.5 \%)$ \\
\hline Hypertension after 35 year old & $22(68.8 \%)$ & $5(62.5 \%)$ \\
\hline \multicolumn{3}{|l|}{ Antihypertensive medication ( $\mathrm{N}, \%$ ) } \\
\hline ARB or ACE inhibitors & $19(59.4 \%)$ & $5(62.5 \%)$ \\
\hline Calcium channel blockers & $12(37.5 \%)$ & $3(37.5 \%)$ \\
\hline $\begin{array}{l}\text { History of gross hematuria from } \\
\text { ruptured cysts }(N, \%)\end{array}$ & $4(12.5 \%)$ & $1(12.5 \%)$ \\
\hline History of infected cysts ( $N, \%)$ & $7(21.9 \%)$ & $2(25 \%)$ \\
\hline Fasting blood sugar (mg/dL) & $96.6 \pm 9.0$ & $95.4 \pm 8.6$ \\
\hline LDL cholesterol (mg/dL) & $109.2 \pm 28.1$ & $110.3 \pm 29.8$ \\
\hline Serum uric acid (mg/dL) & $6.01 \pm 1.26$ & $6.11 \pm 1.29$ \\
\hline Serum albumin $(g / L)$ & $37.7 \pm 6.9$ & $37.6 \pm 5.1$ \\
\hline Serum creatinine (mg/dL) & $0.94 \pm 0.39$ & $0.96 \pm 0.36$ \\
\hline eGFR: CKD-EPI (mL/min/1.73 m²) & $80.3 \pm 24.9$ & $79.8 \pm 24.8$ \\
\hline $\operatorname{mGFR}\left(\mathrm{mL} / \mathrm{min} / 1.73 \mathrm{~m}^{2}\right)$ & $83.8 \pm 26.1$ & $80.7 \pm 24.0(N=2)$ \\
\hline $\mathrm{htTKV}(\mathrm{mL} / \mathrm{m})$ & $681.0 \pm 476.3$ & $670.9 \pm 452.7(N=3)$ \\
\hline \multicolumn{3}{|l|}{ Mayo imaging classification ${ }^{a}$} \\
\hline $1 \mathrm{~A}(\mathrm{~N}, \%)$ & $8(25 \%)$ & $1(33.3 \%)$ \\
\hline $1 \mathrm{~B}(\mathrm{~N}, \%)$ & $11(34.4 \%)$ & $1(33.3 \%)$ \\
\hline $1 \mathrm{C}(\mathrm{N}, \%)$ & $9(28.1 \%)$ & $1(33.3 \%)$ \\
\hline $1 \mathrm{D}(\mathrm{N}, \%)$ & $4(12.5 \%)$ & 0 \\
\hline $1 \mathrm{E}(\mathrm{N}, \%)$ & 0 & 0 \\
\hline $\begin{array}{l}\text { 24-hr urine protein } \\
\left(\mathrm{mg} / \text { day } / 1.73 \mathrm{~m}^{2}\right)\end{array}$ & $205.5 \pm 174.0$ & $194.5 \pm 177.5(N=2)$ \\
\hline $\begin{array}{l}\text { 24-hr urine albumin } \\
\left(\mathrm{mg} / \text { day/1.73 } \mathrm{m}^{2}\right)\end{array}$ & $79.0 \pm 120.2$ & $77.1 \pm 85.2(N=2)$ \\
\hline
\end{tabular}

Data presented as mean \pm SD unless otherwise indicated $B M I$ body mass index; SBP systolic blood pressure; $D B P$ diastolic blood pressure; $A R B$ angiotensin receptor blocker; $A C E$ inhibitor angiotensin converting enzyme inhibitor; $L D L$ cholesterol low density lipoprotein cholesterol, eGFR estimated glomerular filtration rate; CKD-EPI Chronic Kidney Disease Epidemiology Collaboration; $m G F R$ measured glomerular filtration rate; htTKV height-adjusted total kidney volume

aeference [31] 
baseline characteristics and contemporaneous measurements of the patients. There is no statistical difference in clinical parameters between complete and incomplete study group. In the complete study group, the mean age was $56.3 \pm 12.7$ years old. The mean baseline systolic and diastolic blood pressure was $130.6 \pm 15.5$ and $79.2 \pm 8.7 \mathrm{mmHg}$, respectively. Out of 32 patients, 24 (75\%) had hypertension and $2(6.3 \%)$ developed hypertension before the age of 35 years old. All hypertensive patients were advised to take angiotensin receptor blockers (ARBs) or angiotensin converting enzyme inhibitors (ACE inhibitors) unless they had an allergy or intolerance to them. Of the 24 hypertensive patients, 19 (59.4\%) took ARBs or ACE inhibitors and 12 (37\%) had calcium channel blockers. Four (12.5\%) patients had a history of gross hematuria and 7 (21.9\%) experienced a condition of infected cysts. No patient had undergone cyst aspiration or sclerotherapy for large kidney cysts during the study period.

The mean $( \pm \mathrm{SD})$ baseline serum creatinine was $0.94 \pm$ $0.39 \mathrm{mg} / \mathrm{dL}$. The mean initial eGFR by CKD-EPI was $80.3 \pm 24.9 \mathrm{~mL} / \mathrm{min} / 1.73 \mathrm{~m}^{2}$. The mean initial $\mathrm{mGFR}$ was $83.8 \pm 26.1 \mathrm{~mL} / \mathrm{min} / 1.73 \mathrm{~m}^{2}$. The mean change in eGFR and mGFR per year $(\triangle \mathrm{eGFR} / \mathrm{y} \& \Delta \mathrm{mGFR} / \mathrm{y})$ was $-2.91 \pm$ 2.75 and $-2.99 \pm 2.86 \mathrm{~mL} / \mathrm{min} / 1.73 \mathrm{~m}^{2} /$ year, respectively. The mean percentage change in eGFR and mGFR per year $(\% \Delta \mathrm{eGFR} / \mathrm{y} \& \% \Delta \mathrm{mGFR} / \mathrm{y})$ was $-4.14 \pm 4.87$ and $-4.21 \pm$ $4.45 \% /$ year, respectively (Fig. 1). The mean initial TKV was $1083.4 \pm 738.7 \mathrm{~mL}$ and the mean initial htTKV was $681.0 \pm 476.3 \mathrm{~mL} / \mathrm{m}$. The mean change in htTKV per year $(\Delta \mathrm{htTKV} / \mathrm{y})$ was $38.1 \pm 61.9 \mathrm{~mL} / \mathrm{m} /$ year and the mean percentage change in htTKV per year $(\% \Delta \mathrm{htTKV} / \mathrm{y})$ was $4.77 \pm 3.90 \%$ /year (Fig. 1). According to the Mayo clinic ADPKD classification scheme [31], patients were divided into 5 groups by htTKV and age (1A to $1 \mathrm{E}$, low risk to high risk); most patients in this study were identified as group 1A, 1B and 1C (Table 1). All of the patients remained in the same class over the duration of the study. The mean 24-hr urine protein and 24-hr urine albumin were $205.5 \pm$ 174.0 and $76.2 \pm 111.2 \mathrm{mg} /$ day $/ 1.73 \mathrm{~m}^{2}$, respectively.

\section{Comparison between eGFR and mGFR for clinical correlation}

Renal function evaluated by eGFR had a strong association with mGFR $(r=0.718, p<0.001)$. The equation was as follows: $y=0.933 x+0.11$ (Fig. 2). However, when compared the clinical correlation between eGFR and mGFR, we found that mGFR had a better association with clinical parameters. Table 2 demonstrated that initial mGFR had a better association with clinical parameters compared to initial eGFR. Initial mGFR was significantly associated with 4 clinical parameters, including age, initial htTKV, 24-hr urine protein and 24-hr urine albumin, while initial eGRF was significantly associated with only 2 clinical parameters (age and initial htTKV). Moreover, in these two significant clinical parameters, initial mGFR had a better association compared to initial eGFR ( $p$ age: 0.015 vs. $0.041, p$ initial htTKV: $<0.001$ vs. 0.003$)$. Table 3 also confirmed the finding that mGFR had a better association with clinical parameters compared to eGFR. Table 3 demonstrated that the percentage change in measured GFR per year $(\% \Delta \mathrm{mGFR} / \mathrm{y})$ had a better association with clinical parameters compared to the percentage change in estimated GFR per year (\% $\Delta \mathrm{eGFR} / \mathrm{y}) . \% \Delta \mathrm{mGFR} / \mathrm{y}$ was significantly associated with 4 clinical parameters, including initial mGFR, 24-hr urine protein, 24-hr urine albumin and percentage change in height-adjusted total kidney volume per year (\% $\Delta \mathrm{htTKV} / \mathrm{y})$, while $\% \triangle \mathrm{eGFR} / \mathrm{y}$ was significantly associated with only one clinical parameter (initial eGFR). Furthermore, in this significant clinical parameter, $\% \Delta \mathrm{mGFR} / \mathrm{y}$ had a better association compared to $\% \Delta \mathrm{eGFR} / \mathrm{y}$ ( $p$ initial mGFR vs. $p$ initial eGFR: 0.006 vs. 0.030$)$.

\section{The association between clinical parameters and initial mGFR}

Since we found that mGFR had a better association with clinical parameter than eGFR did (Tables 2 and 3), we decided to use mGFR to represent renal function of the patients throughout the studies.

Using Pearson's correlation coefficients, the study demonstrated that initial mGFR was significantly inversely correlated with 4 clinical parameters, including age $(r=-0.427, p=0.015)$, initial htTKV $(r=-0.602$, $p<0.001)$, 24-hr urine protein $(r=-0.399, p=0.024)$, and 24-hr urine albumin $(r=-0.410, p=0.020)$ as shown in Table 2 . It should be noted that when using eGFR instead of mGFR, the association between initial eGFR and 2 clinical parameters including, 24-hr urine protein and 24-hr urine albumin were not statistically significant (Table 2).

Further investigation by using multivariate regression analysis revealed that initial mGFR was significantly, independently, and inversely correlated only with initial htTKV $(p<0.001)$ and age $(p=0.016)$, Table 2 .

Figure 3 demonstrates the relationship between mGFR and htTKV in 32 ADPKD patients over the study period using all data points of each patient. It showed that mGFR was significantly inversely correlated with htTKV. The equation was as follows: $y=-0.032 x+104.06$, $r=-0.599, p<0.001$.

\section{The association between clinical parameters and the percentage change in mGFR per year}

The $\% \Delta \mathrm{mGFR} / \mathrm{y}$ was significantly correlated with initial $\operatorname{mGFR}(r=0.476, p<0.006)$ and inversely correlated with other 3 clinical parameters, including 24-hr urine protein $(r=-0.419, p=0.017)$, 24-hr urine albumin $(r=-$ 0.497, $p=0.004)$ and \% $\Delta$ htTKV/y $(r=-0.501, p=0.004)$ as shown in Table 3 . When using eGFR instead of 


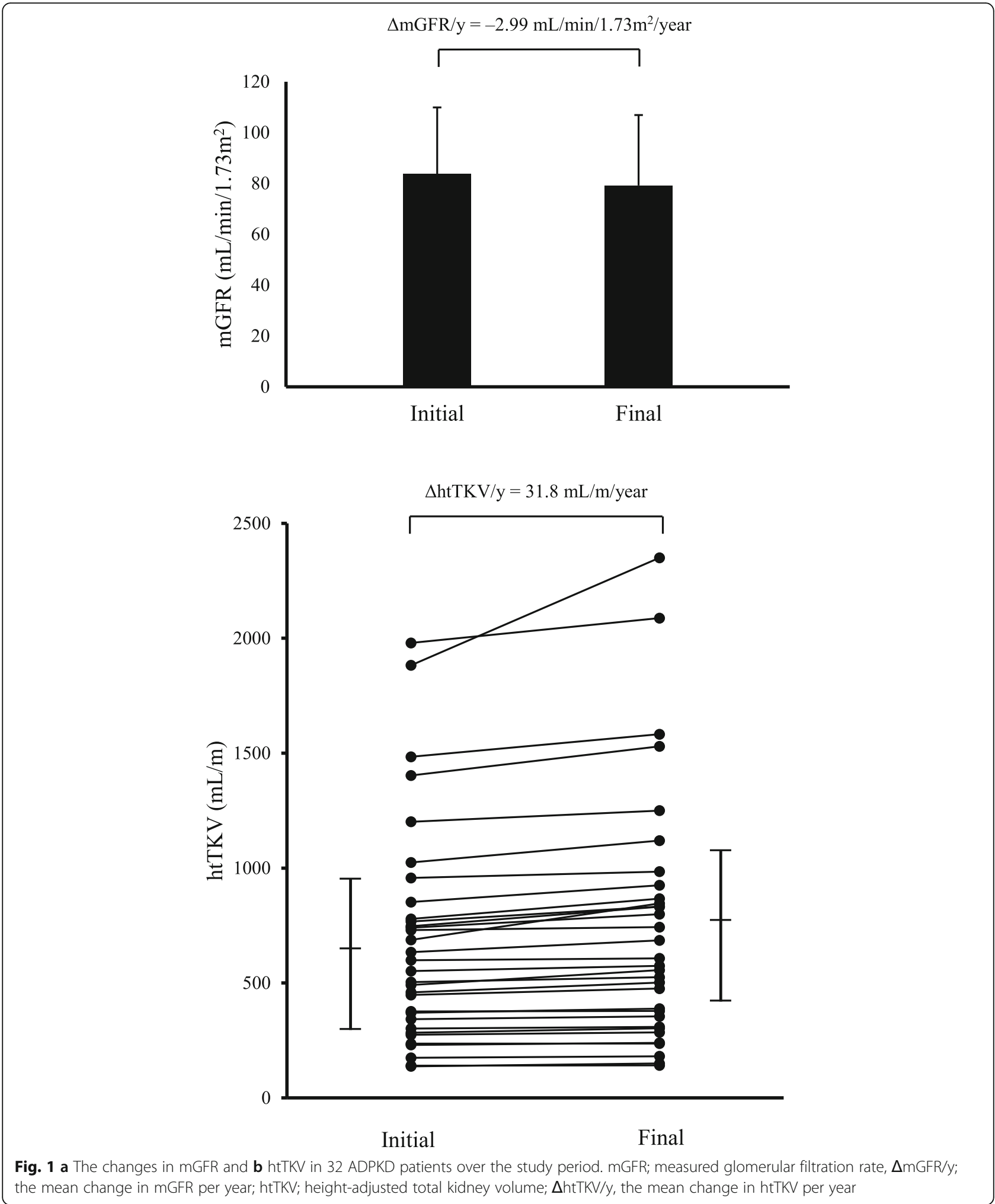

mGFR, the association between the \% $\Delta \mathrm{eGFR} / \mathrm{y}$ and 3 clinical parameters including, 24-hr urine protein, 24-hr urine albumin, and $\% \Delta \mathrm{htTKV} / \mathrm{y}$ were not statistically significant (Table 3).
Further multivariate analysis showed that the $\% \Delta$ mGFR/ $\mathrm{y}$ was significantly, independently, and inversely correlated only with the $\% \Delta$ htTKV/y $(p=0.032)$ and 24-hr urine albumin $(p=0.034)$, Table 3. 


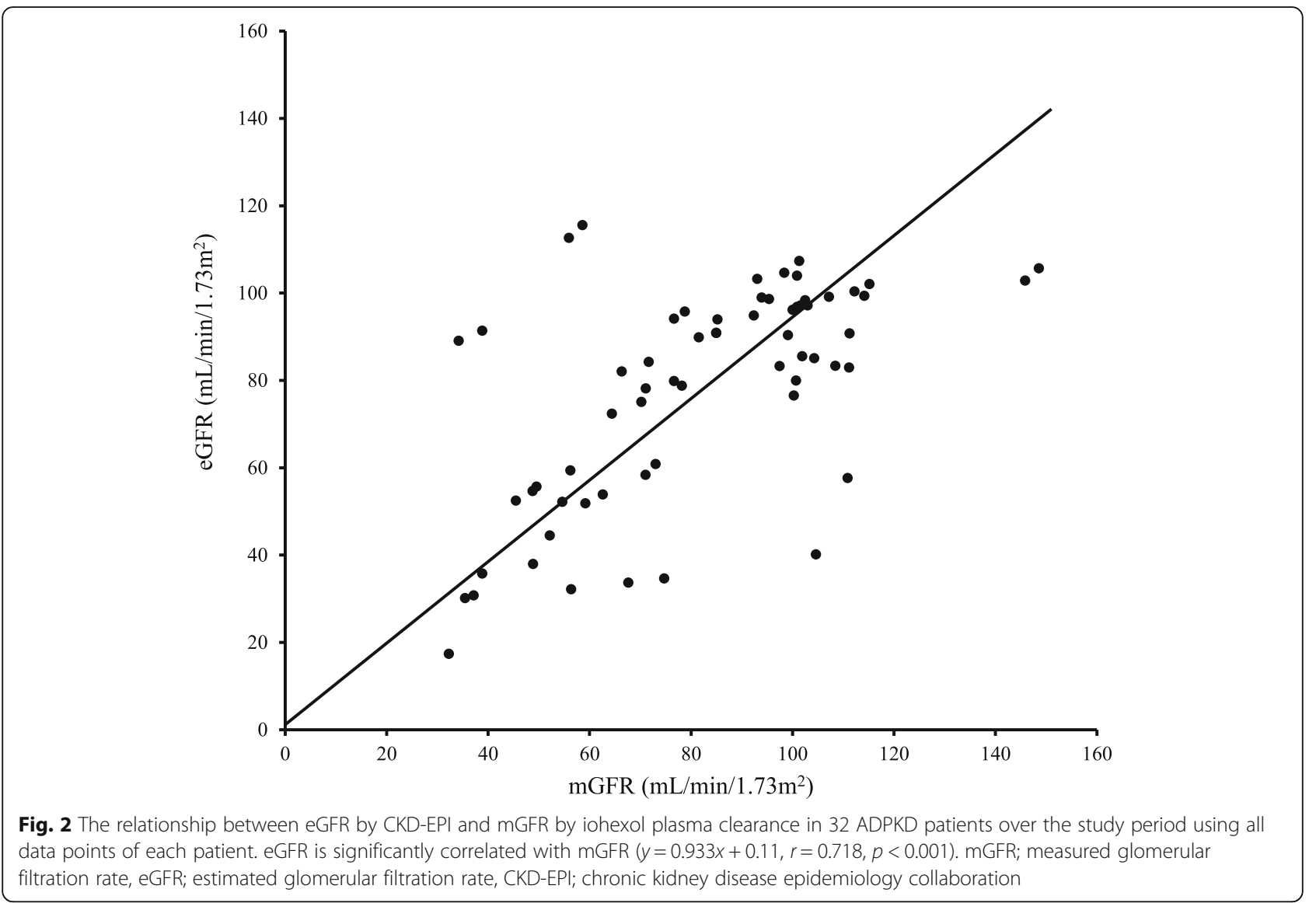

Table 2 The association of initial eGFR and initial mGFR on different clinical parameters

\begin{tabular}{|c|c|c|c|c|c|c|c|c|c|}
\hline \multirow{3}{*}{$\begin{array}{l}\text { Clinical parameters } \\
(N=32)\end{array}$} & \multirow{2}{*}{\multicolumn{3}{|c|}{$\begin{array}{l}\text { initial eGFR } \\
\text { Univariate analysis }\end{array}$}} & \multicolumn{6}{|c|}{ initial mGFR } \\
\hline & & & & \multicolumn{3}{|c|}{ Univariate analysis } & \multicolumn{3}{|c|}{ Multivariate analysis } \\
\hline & $r$ & $95 \% \mathrm{Cl}$ & $p$ & $r$ & $95 \% \mathrm{Cl}$ & $p$ & $r$ & $95 \% \mathrm{Cl}$ & $p$ \\
\hline Age (year) & -0.364 & -1.390 to -0.032 & $0.041^{*}$ & -0.427 & -1.568 to -0.184 & $0.015^{*}$ & -0.346 & -1.279 to -0.142 & $0.016^{*}$ \\
\hline $\mathrm{BMI}\left(\mathrm{Kg} / \mathrm{m}^{2}\right)$ & -0.164 & -4.198 to 1.609 & 0.370 & -0.041 & -3.428 to 2.743 & 0.822 & & & \\
\hline Mean SBP (mmHg) & -0.144 & -0.827 to 0.361 & 0.430 & -0.183 & -0.929 to 0.310 & 0.316 & & & \\
\hline Mean DBP (mmHg) & -0.009 & -1.088 to 1.037 & 0.961 & -0.083 & -1.361 to 0.862 & 0.650 & & & \\
\hline $\mathrm{LDL}(\mathrm{mg} / \mathrm{dL})$ & 0.111 & -0.230 to 0.426 & 0.546 & -0.058 & -0.399 to 0.292 & 0.754 & & & \\
\hline Serum uric acid (mg/dL) & -0.447 & -15.438 to -2.240 & 0.100 & -0.264 & -12.936 to 1.997 & 0.145 & & & \\
\hline Serum albumin $(g / L)$ & -0.060 & -1.541 to 1.112 & 0.743 & -0.176 & -1.514 to 1.273 & 0.861 & & & \\
\hline Initial htTKV (mL/m) & -0.510 & -0.043 to -0.010 & $0.003^{*}$ & -0.602 & -0.049 to 0.017 & $<0.001^{*}$ & -0.551 & -0.045 to -0.015 & $<0.001^{*}$ \\
\hline $\begin{array}{l}\text { 24-hr urine protein } \\
\left(\mathrm{mg} / \text { day/1.73 } \mathrm{m}^{2}\right)\end{array}$ & -0.326 & -0.097 to -0.004 & 0.068 & -0.399 & -0.111 to -0.009 & $0.024^{*}$ & -0.157 & -0.096 to 0.049 & 0.513 \\
\hline $\begin{array}{l}\text { 24-hr urine albumin } \\
\left(\mathrm{mg} / \text { day/1.73 } \mathrm{m}^{2}\right)\end{array}$ & -0.332 & -0.153 to 0.004 & 0.063 & -0.410 & -0.176 to -0.016 & $0.020^{*}$ & -0.021 & -0.112 to 0.122 & 0.932 \\
\hline
\end{tabular}

* indicates statistical significance $(P<0.05)$

eGFR estimated glomerular filtration rate; $m G F R$ measured glomerular filtration rate; $B M I$ body mass index; SBP systolic blood pressure; DBP diastolic blood pressure; $L D L$ cholesterol, low density lipoprotein cholesterol; $h t T K V$ height-adjusted total kidney volume 
Table 3 The association of the percentage change in estimated GFR per year ( $\% \Delta \mathrm{eGFR} / \mathrm{y}$ ) and the percentage change in measured GFR per year (\% $\Delta \mathrm{mGFR} / \mathrm{y})$ on different clinical parameters

\begin{tabular}{|c|c|c|c|c|c|c|c|c|c|}
\hline \multirow{3}{*}{$\begin{array}{l}\text { Clinical parameters } \\
(N=32)\end{array}$} & \multirow{2}{*}{\multicolumn{3}{|c|}{$\frac{\% \Delta \mathrm{eGFR} / \mathrm{y}}{\text { Univariate analysis }}$}} & \multicolumn{6}{|c|}{$\% \Delta \mathrm{mGFR} / \mathrm{y}$} \\
\hline & & & & \multicolumn{3}{|c|}{ Univariate analysis } & \multicolumn{3}{|c|}{ Multivariate analysis } \\
\hline & $\bar{r}$ & $95 \% \mathrm{Cl}$ & $p$ & $\bar{r}$ & $95 \% \mathrm{Cl}$ & $p$ & $r$ & $95 \% \mathrm{Cl}$ & $p$ \\
\hline Age (year) & -0.082 & -0.173 to 0.111 & 0.656 & -0.100 & -0.165 to 0.095 & 0.587 & & & \\
\hline BMI $\left(\mathrm{Kg} / \mathrm{m}^{2}\right)$ & -0.194 & -0.863 to 0.266 & 0.288 & -0.137 & -0.715 to 0.328 & 0.454 & & & \\
\hline Mean SBP (mmHg) & 0.093 & -0.088 to 0.146 & 0.612 & -0.148 & -0.149 to 0.064 & 0.418 & & & \\
\hline Mean DBP $(\mathrm{mmHg})$ & 0.052 & -0.179 to 0.236 & 0.778 & -0.131 & -0.255 to 0.122 & 0.476 & & & \\
\hline Initial eGFR (mL/min/1.73 m²) & 0.385 & 0.008 to 0.142 & $0.030^{*}$ & - & - & - & & & \\
\hline Initial mGFR (mL/min/1.73 $\left.\mathrm{m}^{2}\right)$ & - & - & - & 0.476 & 0.025 to 0.137 & $0.006^{*}$ & 0.265 & -0.013 to 0.103 & 0.123 \\
\hline Initial htTKV (mL/m) & 0.126 & -0.002 to 0.005 & 0.493 & -0.282 & -0.006 to 0.001 & 0.117 & & & \\
\hline $\begin{array}{l}\text { 24-hr urine protein } \\
\left(\mathrm{mg} / \text { day } / 1.73 \mathrm{~m}^{2}\right)\end{array}$ & -0.301 & -0.018 to 0.002 & 0.094 & -0.419 & -0.019 to -0.002 & $0.017^{*}$ & 0.038 & -0.012 to 0.014 & 0.881 \\
\hline $\begin{array}{l}\text { 24-hr urine albumin } \\
\left(\mathrm{mg} / \text { day/1.73 } \mathrm{m}^{2}\right)\end{array}$ & -0.205 & -0.025 to 0.007 & 0.261 & -0.497 & -0.033 to -0.007 & $0.004^{*}$ & -0.357 & -0.027 to -0.001 & $0.034^{*}$ \\
\hline$\% \Delta$ htTKV/y (\%/year) & -0.254 & -0.767 to 0.133 & 0.161 & -0.501 & -0.941 to -0.203 & $0.004^{*}$ & -0.363 & -0.791 to -0.039 & $0.032^{*}$ \\
\hline
\end{tabular}

* indicates statistical significance $(P<0.05)$

$\% \triangle e G F R / y$ percentage change in estimated GFR per year, \% $\triangle m G F R / y$ percentage change in measured GFR per year; $B M I$ body mass index; SBP systolic blood pressure; DBP diastolic blood pressure; $h t T K V$ height-adjusted total kidney volume; $\% \Delta h t T K V / y$ percentage change in height-adjusted total kidney volume per year

Figure 4 demonstrates the relationship between the $\% \Delta \mathrm{mGFR} / \mathrm{y}$ and the $\% \Delta \mathrm{htTKV} / \mathrm{y}$. The $\% \Delta \mathrm{mGFR} / \mathrm{y}$ was significantly inversely correlated with the $\% \Delta \mathrm{htTKV} / \mathrm{y}$. The equation was as follows: $y=-0.572 x-1.486, r=-0.501$, $p=0.004$.
For purpose of clinical application, univariate regression analysis was utilized to study the relationship between the $\% \Delta \mathrm{mGFR} / \mathrm{y}$ and the $\% \Delta \mathrm{htTKV} / \mathrm{y}$. It was found that there was an association between a $5 \%$ change in $\% \Delta \mathrm{htTKV} / \mathrm{y}$ and a $5 \%$ change in $\% \Delta \mathrm{mGFR} / \mathrm{y}$. In this study, no patients

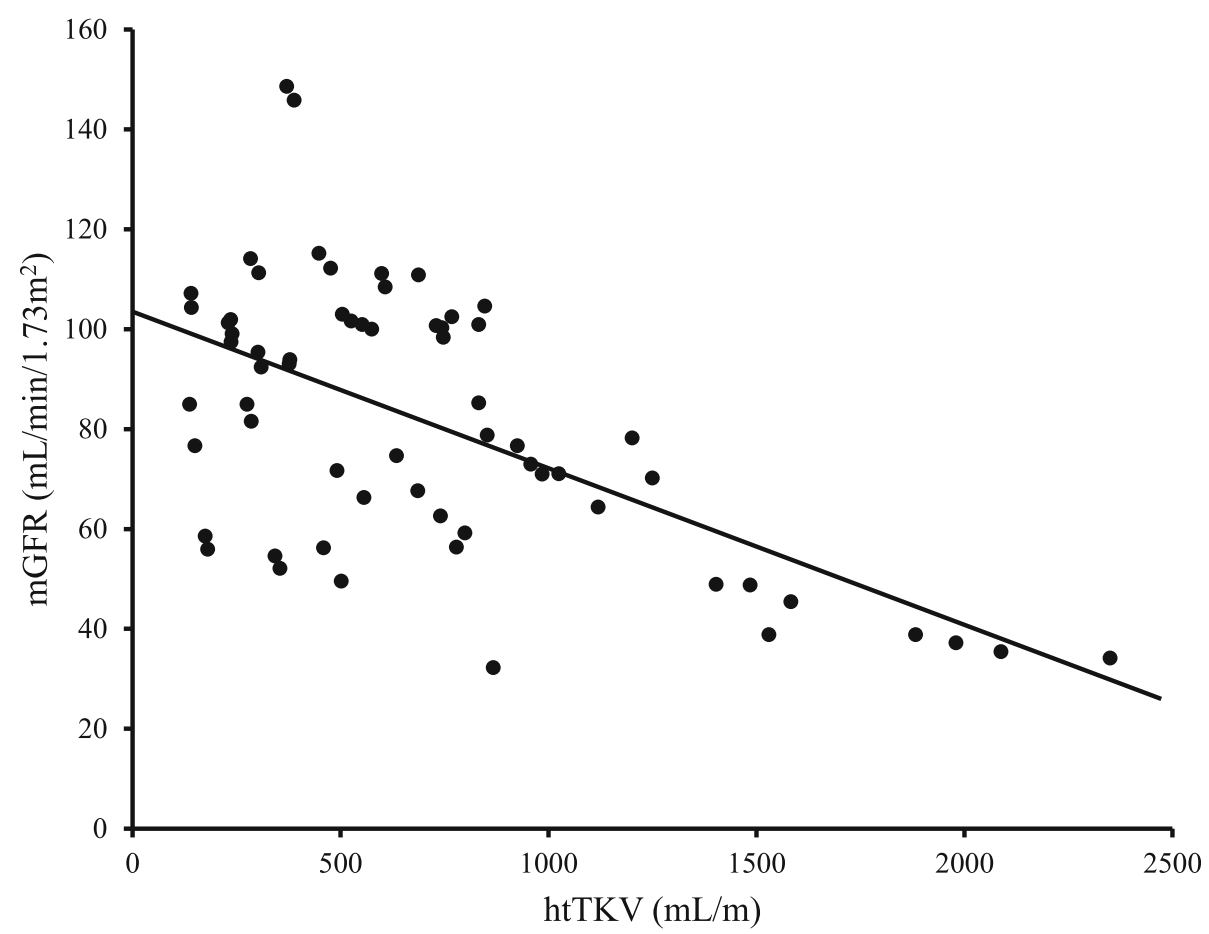

Fig. 3 The relationship between mGFR and htTKV in 32 ADPKD patients over the study period using all data points of each patient. mGFR is significantly inversely correlated with htTKV $(y=-0.032 x+104.06, r=-0.599, p<0.001)$. mGFR; mearsured glomerular filtration rate, htTKV; heightadjusted total kidney volume 


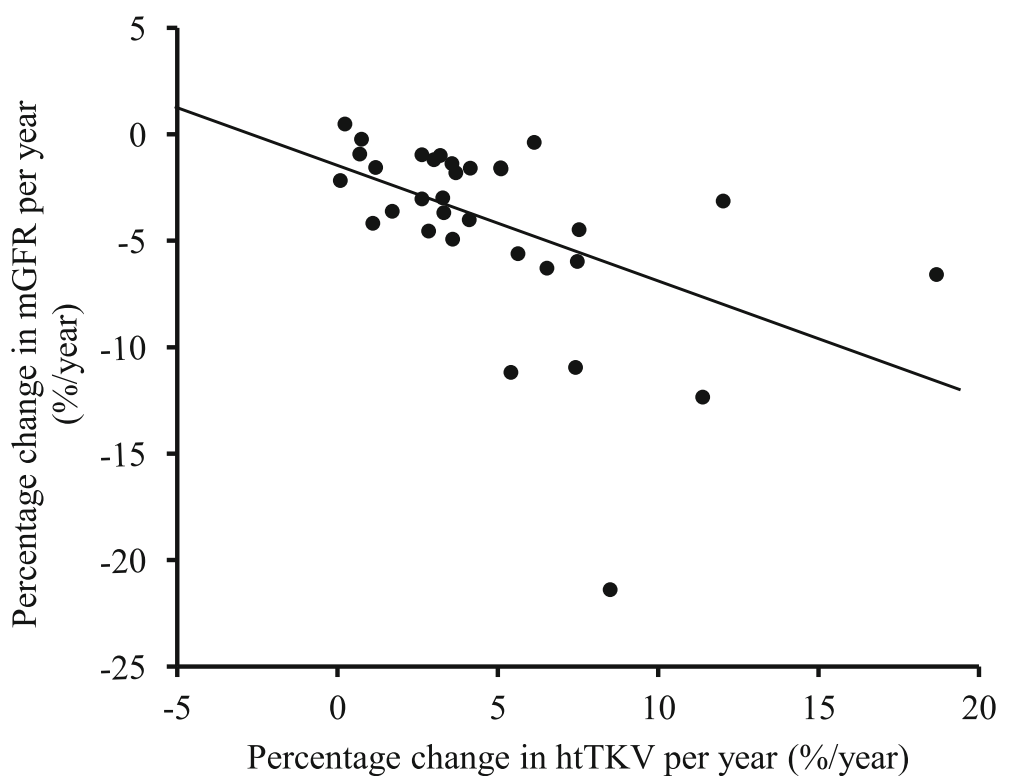

Fig. 4 The relationship between the percentage change in mGFR per year (\% $\Delta \mathrm{mGFR} / \mathrm{y})$ and the percentage change in htTKV per year (\% $\Delta \mathrm{htTKV/}$ y) in 32 ADPKD patients over the study period. The $\% \Delta$ mGFR/y is significantly inversely correlated with the $\% \Delta$ tTKV/y $(y=-0.572 x-1.486, r=-$ $0.501, p=0.004$.). mGFR; measured glomerular filtration rate, htTKV; height-adjusted total kidney volume; \% $\Delta \mathrm{mGFR} / \mathrm{y}$, percentage change in mGFR per year; $\% \Delta h t T K V / y$, percentage change in htTKV per year

who had a $\% \Delta$ htTKV/y of less than $5 \%$ developed a $\% \Delta \mathrm{mGFR} / \mathrm{y}$ of more than $5 \%$. By contrast, patients who had a $\% \Delta \mathrm{htTKV} / \mathrm{y}$ of more than or equal to $5 \%$ had a high prevalence $(61.5 \%)$ to develop a $\% \Delta \mathrm{mGFR} / \mathrm{y}$ of more than $5 \%, p=0.003$.

In the case of albuminuria, the ROC curve was used to find the cutting point of 24-hr urine albumin to predict the change in $\% \Delta \mathrm{mGFR} / \mathrm{y}$ of $5 \%$. We found that $24-\mathrm{hr}$ urine albumin at $30 \mathrm{mg} /$ day had a sensitivity of $62.5 \%$ and specificity of $79.2 \%$ to predict this change. In this study, patients who had a 24-hr urine albumin of less than $30 \mathrm{mg} /$ day/1.73 $\mathrm{m}^{2}$ were less likely (13.0\%) to develop a $\% \triangle \mathrm{mGFR} / \mathrm{y}$ of more than $5 \%$. On the other hand, patients who had a 24-hr urine albumin more than or equal to $30 \mathrm{mg} /$ day $/ 1.73 \mathrm{~m}^{2}$ had a higher chance $(55.6 \%)$ to develop a $\% \Delta \mathrm{mGFR} / \mathrm{y}$ of more than $5 \%, p=0.023$.

\section{The association between clinical parameters and the} percentage change in htTKV per year $(\% \Delta \mathrm{htTKV} / \mathrm{y})$

The $\% \Delta \mathrm{htTKV} / \mathrm{y}$ was significantly correlated with initial htTKV $(r=0.420, p=0.017)$ and 24-hr urine albumin $(r=0.384, p=0.030)$ as shown in Table 4. Multivariate regression analysis showed that $\% \Delta \mathrm{htTKV} / \mathrm{y}$ was significantly and independently correlated only with htTKV $(p=0.017)$, Table 4 . The equation was as follows: $y=0.003 x+0.429, r=0.420, p=0.017$.

\section{The effect of ARBs/ACE inhibitors on clinical parameters} In this study, there were 19 patients using at least one ARB or ACE inhibitor for treatment of hypertension. The other
13 (5 with hypertension on calcium channel blockers and 8 without hypertension) patients did not use ARBs or ACE inhibitors. Clinical parameters of ADPKD patients using ARBs/ACE inhibitors versus without ARBs/ACE inhibitors were shown in Table 5 . There was no difference in blood pressure between the two groups. However, the mean 24$\mathrm{hr}$ urine albumin was significantly lower in patients using ARBs/ACE inhibitors group (38.2 \pm 57.4 vs. $131.8 \pm 146.1$, $\mathrm{mg} /$ day $/ 1.73 \mathrm{~m}^{2}, p=0.045$ ). Likewise, the mean 24-hr urine protein also had a tendency to be lower in patients using ARBs/ACE inhibitors group ( $150.5 \pm 81.6$ vs. $285.8 \pm 237.6$, $\mathrm{mg} /$ day $/ 1.73 \mathrm{~m}^{2}, p=0.069$ ), although this did not reach a statistically significant $p$-value. In addition, the mean $24-\mathrm{hr}$ urine sodium tended to be higher in patients using ARBs/ ACE inhibitors group (150.7 \pm 25.9 vs. $135.2 \pm 20.4$, mEq/ day, $p=0.070$ ), but also not statistically significant. The $\% \Delta \mathrm{htTKV} / \mathrm{y}$ was not significantly different between the two groups (4.62 \pm 3.43 vs. $4.98 \pm 4.63$, \%/year, $p=0.812$ ), and neither was the $\% \Delta \mathrm{mGFR} / \mathrm{y}(-4.03 \pm 3.59$ vs. $-4.48 \pm$ 5.63 , \%/year, $p=0.804$ ).

\section{Discussion}

Evaluation of renal function is a crucial part in assessing kidney disease, especially in ADPKD patients whose kidney functions remain stable for years, followed by a sharp decline in GFR once a critical renal size is reached $[6,7]$. Assessment of renal function by estimated GFR using CKD-EPI and MDRD has been shown to have wide and unpredictable deviation of estimations compared to the measured GFR changes in ADPKD patients 
Table 4 The association of the percentage change in height-adjusted total kidney volume per year (\% $\Delta$ htTKV/y) and different clinical parameters

\begin{tabular}{|c|c|c|c|c|c|c|}
\hline \multirow{3}{*}{$\begin{array}{l}\text { Clinical parameters } \\
(N=32)\end{array}$} & \multicolumn{6}{|c|}{$\% \Delta$ htTKV/y } \\
\hline & \multicolumn{3}{|c|}{ Univariate analysis } & \multicolumn{3}{|c|}{ Multivariate analysis } \\
\hline & $r$ & $95 \% \mathrm{Cl}$ & $p$ & $r$ & $95 \% \mathrm{Cl}$ & $p$ \\
\hline Age (year) & 0.073 & -0.092 to 0.136 & 0.692 & & & \\
\hline $\mathrm{BMI}\left(\mathrm{Kg} / \mathrm{m}^{2}\right)$ & 0.096 & -0.340 to 0.578 & 0.600 & & & \\
\hline Mean SBP (mmHg) & 0.115 & -0.064 to 0.122 & 0.532 & & & \\
\hline Mean DBP (mmHg) & 0.324 & -0.013 to 0.302 & 0.071 & & & \\
\hline Initial eGFR (mL/min/1.73 m²) & -0.158 & -0.082 to 0.033 & 0.387 & & & \\
\hline Initial mGFR (mL/min/1.73 m²) & -0.333 & -0.102 to 0.003 & 0.062 & & & \\
\hline Initial htTKV (mL/m) & 0.420 & 0.001 to 0.006 & $0.017^{*}$ & 0.420 & 0.001 to 0.006 & $0.017^{*}$ \\
\hline 24-hr urine protein (mg/day/1.73 m²) & 0.349 & 0.000 to 0.016 & 0.050 & & & \\
\hline 24-hr urine albumin (mg/day/1.73 $\left.\mathrm{m}^{2}\right)$ & 0.384 & 0.001 to 0.026 & $0.030^{*}$ & 0.219 & -0.006 to 0.022 & 0.276 \\
\hline
\end{tabular}

* indicates statistical significance $(P<0.05)$

$B M I$ body mass index; SBP systolic blood pressure; DBP diastolic blood pressure; eGFR estimated glomerular filtration rate; $m G F R$ measured glomerular filtration rate; $h t T K V$ height-adjusted total kidney volume; $\% \Delta h t T K V / y$ percentage change in height-adjusted total kidney volume per year

[32]. The authors in the former study stated that oneyear GFR changes estimated by both prediction formulas failed to correlate, to an appreciable extent, with measured changes and suggested that these surrogate outcome variables were not appropriate to assess progression of ADPKD and response to treatment in research and clinics. In our studies, we also reaffirmed these results - the measured GFR by iohexol had a better correlation with clinical parameters compared to eGFR (CKD-EPI). Thus, in this study we used the mGFR by iohexol to represent renal function in our cohort of ADPKD patients. From our understanding, this is the first study to evaluate renal function by measured GFR using iohexol in an Asian ADPKD population.

The mGFR change in ADPKD patients in our studies was $-2.99 \mathrm{~mL} / \mathrm{min} / 1.73 \mathrm{~m}^{2} /$ year, which was comparable to GFR change measured by iothalamate clearance -2.79 $\mathrm{mL} / \mathrm{min} / 1.73 \mathrm{~m}^{2} /$ year in the US population [33]. This is likely due to the initial GFR (this study 83.8 VS US 91.4 $\mathrm{mL} / \mathrm{min} / 1.73 \mathrm{~m}^{2}$ ) and initial TKV (this study $1083 \mathrm{VS}$ US $1193 \mathrm{~mL}$ ) being comparable between our and the US population. When compared with the Japanese ADPKD population, the estimated GFR change per year in Japanese ADPKD was $-2.8 \mathrm{~mL} / \mathrm{min} / 1.73 \mathrm{~m}^{2} /$ year by using the MDRD Japanese equation [34]. The GFR change in our studies was also comparable to the Japanese population. However, the initial estimated GFR of $55.3 \mathrm{~mL} /$ $\mathrm{min} / 1.73 \mathrm{~m}^{2}$ and initial TKV of $1595 \mathrm{~mL}$ in Japanese studies were quite different to our population. This discrepancy in results may be explained by the difference in method used to evaluate GFR between the Japanese and our population (estimated GFR by Japanese MDRD equation and measured GFR by iohexol).

The calculated annual kidney growth rate in our study was $4.8 \%$ (baseline TKV of $1083 \mathrm{~mL}$ ) which was in agreement with the previous reported of $4 \%$ with baseline TKV of $1194 \mathrm{~mL}$ [33], and $5.2 \%$ with baseline TKV of $1060 \mathrm{~mL}$ [14]. In addition, the results of our studies showed that the percentage change in htTKV per year was significantly correlated solely with initial htTKV (Table 4). This finding was similar to the results of previous studies which reported that the baseline total

Table 5 Clinical parameters of patients on ARBs/ACE inhibitors versus without ARBs/ACE inhibitors

\begin{tabular}{llll}
\hline Clinical parameters & On ARBs/ACE inhibitors $(\boldsymbol{N}=\mathbf{1 9})$ & No ARBs/ACE inhibitors $(\boldsymbol{N}=\mathbf{1 3})$ & $\boldsymbol{P}$ Value \\
\hline SBP $(\mathrm{mmHg})$ & $130.9 \pm 15.6$ & $130.2 \pm 15.9$ & 0.908 \\
DBP $(\mathrm{mmHg})$ & $79.5 \pm 9.1$ & $78.8 \pm 8.5$ & 0.825 \\
24-hr urine protein $\left(\mathrm{mg} /\right.$ day/1.73 $\left.\mathrm{m}^{2}\right)$ & $150.5 \pm 81.6$ & $285.8 \pm 237.6$ & 0.069 \\
24-hr urine albumin (mg/day/1.73 $\left.\mathrm{m}^{2}\right)$ & $38.2 \pm 57.4$ & $131.8 \pm 146.1$ & $0.045^{*}$ \\
24-hr urine sodium (mEq/day) & $150.7 \pm 25.9$ & $135.2 \pm 20.4$ & 0.070 \\
\% $\Delta$ htTKV/y (\%/year) & $4.62 \pm 3.43$ & $4.98 \pm 4.63$ & 0.812 \\
\% $\Delta$ mGFR/y (\%/year) & $-4.03 \pm 3.59$ & $-4.48 \pm 5.63$ & 0.804 \\
\hline
\end{tabular}

Data presented as mean \pm SD

* indicates statistical significance $(P<0.05)$

$A R B$ angiotensin receptor blocker; $A C E$ inhibitor angiotensin converting enzyme inhibitor; SBP systolic blood pressure; $D B P$ diastolic blood pressure; \% $\triangle$ htTKV/

$y$ percentage change in height-adjusted total kidney volume per year; \% $\triangle m G F R / y$ percentage change in measured GFR per year 
kidney volume predicted the subsequent rate of increase in volume [14], Because renal growth is a continuous and relatively constant process, patients with the largest kidneys at a certain age should have the fastest rate of kidney enlargement. The results of CRISP have shown that the kidney growth occurs in an exponential fashion and the growth of kidneys in patients with ADPKD is primarily the result of growth of cysts. ADPKD disease progression was due to the increasing in size of an extremely large number of renal cysts, which causes enlargement of the whole kidney $[14,35,36]$. The larger kidneys are likely to be associated with more complications such as hypertension, pain, hematuria, hemorrhagic cysts, infected cysts, and the decline in renal function.

The relationship between the total kidney volume and renal function was well demonstrated in our studies (Table 2 and Fig. 3). Our studies have shown that the initial mGFR is significantly, independently, and inversely associated with patient's age and initial htTKV and the latter has a stronger association. Our results were similar with the results reported in previous studies that showed the inverse correlation of kidney volume and renal function [14, 36]. Moreover, our studies also demonstrated that the percentage change of mGFR per year was significantly, independently, and inversely associated with the percentage change in height-adjusted total kidney volume per year and 24-hr urine albumin (Table 3 and Fig. 4). The inverse association between the change in renal function and the change in total kidney volume was well demonstrated. Several studies have shown that the rate of increase in TKV were highly variable and that patients with high rates of growth were more likely to suffer serious declines in GFR than those who showed a slower growth rate [14, 36, 37]. The results of our studies also demonstrated that there was an association between a $5 \%$ change in TKV per year and a $5 \%$ change in the mGFR per year. In our population, no patients who had the TKV change of less than $5 \%$ per year developed the mGFR change of more than $5 \%$ per year. By contrast, patients who had a TKV change of more than or equal to $5 \%$ per year had a high prevalence to develop mGFR changes of more than $5 \%$ per year. The sequential measurement of total kidney volume and cyst volume has been suggested as a surrogate marker of disease progression [14, 35-37]. Our findings were in harmony with previous studies.

Albuminuria is well known to be strongly associated with progression in glomerular diseases. A number of studies have shown that in patients with ADPKD, which was classified as a non-glomerular disease, renal progression is also associated with albuminuria $[8,33,34]$. A previous study showed that overt proteinuria (protein > $300 \mathrm{mg} /$ day) was associated with large renal volumes, higher blood pressures, and low creatinine clearances in patients with ADPKD [8]. The studies of ADPKD progression by mGFR using iothalamate clearance showed that patients with greater proteinuria had a significantly larger increase in renal volume and decline in renal function than those with less proteinuria [33]. The findings in our studies that showed the GFR change was significantly, independently, and inversely correlated with 24-hr urine albumin (Table 3) coordinated with the results in previous studies [33]. In addition, our finding by univariate but not by multivariate analysis also suggested that the percentage change in htTKV per year was significantly correlated with 24-hr urine albumin (Table 4). In our population, we found that 24-hr urine albumin at $30 \mathrm{mg} /$ day $/ 1.73 \mathrm{~m}^{2}$ had a good prediction for a $5 \%$ change in GFR per year. Patients who had 24-hr urine albumin of less than $30 \mathrm{mg} /$ day $/ 1.73 \mathrm{~m}^{2}$ were less likely to develop GFR change of more than $5 \%$ per year. On the other hand, patients who had a 24-hr urine albumin more than or equal to $30 \mathrm{mg} /$ day $/ 1.73 \mathrm{~m}^{2}$ had a higher prevalence of developing GFR change of more than $5 \%$ per year.

In this study, the mean 24-hr urine albumin in patients using ARBs/ACE inhibitors was lower than that of patients without ARBs/ACE inhibitors. However, there was no statistically significant difference in the $\% \Delta \mathrm{htTKV} / \mathrm{y}$ and $\% \Delta \mathrm{mGFR} / \mathrm{y}$ between the two groups. The benefits of slowing kidney volume progression did not occur in patients using ARBs/ACE inhibitors in this study. This could be explained by a considerable number of nonhypertensive patients (8/13) in the non ARBs/ACE inhibitors group who may have a better prognosis in the changes of TKV or GFR than hypertensive patients. In addition, the study period may be too short to claim the beneficial effects of these drugs.

Some potential limitations in our study should be acknowledged. Due to the small number of participants, our trial may have had inadequate power to demonstrate the statistical significance of some clinical correlations. Another limitation is the technique used to quantify TKV which we manually measured on the MRI. This gives rise to more variation and inconsistency when compared to other automatic techniques. However, this method was preferred over other techniques such as the fully automated segmentation technique as enlarged ADPKD cysts tend to distort the surrounding anatomical structures of the kidneys, causing differentiation of the anatomical boundaries by automated segmentation almost unfeasible. Hopefully, in the future, a more accurate automated technique will be developed to assess TKV in ADPKD kidneys, allowing for a more consistent measurement.

\section{Conclusions}

Our studies demonstrated that mGFR by iohexol plasma clearance is a more reliable and accurate method than 
eGFR for evaluating GFR changes in the early stages of patients with ADPKD. There is a strong inverse correlation between kidney volume and renal function assessed by mGFR in an Asian ADPKD population. The initial htTKV is a good predictor for kidney volume progression. The $\% \Delta \mathrm{htTKV} / \mathrm{y}$ is a good early surrogate marker for following up the decline in renal function. 24-hr urine albumin is also a good indicator for renal progression.

\begin{abstract}
Abbreviations
ADPKD: Autosomal dominant polycystic kidney disease; ESRD: End stage renal disease; eGFR: Estimated glomerular filtration rate; mGFR: Measured glomerular filtration rate; CKD-EPI: Chronic kidney disease epidemiology collaboration; MDRD: Modification of diet in renal disease; TKV: Total kidney volume; htTKV: Height-adjusted TKV; $\Delta$ htTKV/y: Change in htTKV per year; $\% \Delta h t T K V / y:$ Percentage change in htTKV per year; $\Delta$ eGFR/y: Change in eGFR per year; $\Delta m G F R / y$ : Change in mGFR per year; $\% \Delta e G F R / y$ : Percentage change in eGFR per year; $\% \Delta m G F R / y$ : Percentage change in mGFR per year; CRISP: Consortium of radiologic imaging studies of polycystic kidney disease; UPLC: Ultra performance liquid chromatography
\end{abstract}

\section{Acknowledgements}

Our grateful appreciation is extended to all patients who participated in this study. Additional statistical analyses by Dr. Atiporn Ingsathit and Mrs. Umaporn Udomsubpayakul from the Clinical Epidemiology Unit was greatly appreciated.

\section{Authors' contributions}

Research idea and study design: BP, WT; data acquisition: BP, WT, PW, SB, PK; data analysis/interpretation: BP, WT, PW, SB; statistical analysis: BP, WT; supervision or mentorship: BP. All authors read and approved the final manuscript.

\section{Funding}

This project was supported by The Ramathibodi Research Fund, Mahidol University, Bangkok, Thailand.

\section{Availability of data and materials}

The datasets generated and/or analyzed during the current study are not publicly available due to the institutional policy but are available from the corresponding author on reasonable request.

\section{Declarations}

\section{Ethics approval and consent to participate}

Written informed consent was obtained from all patients. The study was approved by the Institutional Ethics Committee on Human Rights Related to Research Involving Human Subjects of the Ramathibodi Hospital, Mahidol University.

\section{Consent for publication}

Not applicable.

\section{Competing interests}

The authors declare that they have no competing interests.

\section{Author details}

'Division of Nephrology, Department of Medicine, Faculty of Medicine, Ramathibodi Hospital, Mahidol University, 10400 Bangkok, Thailand. 2Department of Diagnostic and Therapeutic Radiology, Faculty of Medicine, Ramathibodi Hospital, Mahidol University, Bangkok, Thailand.
Received: 12 January 2021 Accepted: 6 May 2021

Published online: 15 May 2021

\section{References}

1. Gabow PA. Autosomal dominant polycystic kidney disease. N Engl J Med. 1993;329:332-42.

2. Torres VE, Harris PC, Pirson Y. Autosomal dominant polycystic kidney disease. Lancet. 2007;369:1287-301.

3. Bajwa ZH, Gupta S, Warfield CA, Steinman TI. Pain management in polycystic kidney disease. Kidney Int. 2001;60:1631-44.

4. Grantham JJ. Polycystic kidney disease: from the bedside to the gene and back. Curr Opin Nephrol Hypertens. 2001;10:533-42.

5. Neugarten J, Acharya A, Silbiger SR. Effect of gender on the progression of nondiabetic renal disease: a meta-analysis. J Am Soc Nephrol. 2000;11:319-29.

6. Franz KA, Reubi FC. Rate of functional deterioration in polycystic kidney disease. Kidney Int. 1983;23:526-9.

7. Yu ASL, Shen C, Landsittel DP, Grantham JJ, Cook LT, Torres VE, et al. Longterm trajectory of kidney function in autosomal-dominant polycystic kidney disease. Kidney Int. 2019;95:1253-61.

8. Chapman AB, Johnson AM, Gabow PA, Schrier RW. Overt proteinuria and microalbuminuria in autosomal dominant polycystic kidney disease. J Am Soc Nephrol. 1994;5:1349-54

9. Fick-Brosnahan GM, Belz MM, McFann KK, Johnson AM, Schrier RW. Relationship between renal volume growth and renal function in autosomal dominant polycystic kidney disease: a longitudinal study. Am J Kidney Dis. 2002;39:1127-34.

10. Zheng D, Wolfe M, Cowley BD, Jr., Wallace DP, Yamaguchi T, Grantham JJ. Urinary excretion of monocyte chemoattractant protein-1 in autosomal dominant polycystic kidney disease. J Am Soc Nephrol. 2003;14:2588-95.

11. Gabow PA, Chapman AB, Johnson AM, Tangel DJ, Duley IT, Kaehny WD, et al. Renal structure and hypertension in autosomal dominant polycystic kidney disease. Kidney Int. 1990;38:1177-80.

12. Serra AL, Poster D, Kistler AD, Krauer F, Raina S, Young J, et al. Sirolimus and kidney growth in autosomal dominant polycystic kidney disease. N Engl J Med. 2010;363:820-9.

13. Walz G, Budde K, Mannaa M, Nurnberger J, Wanner C, Sommerer C, et al. Everolimus in patients with autosomal dominant polycystic kidney disease N Engl J Med. 2010;363:830-40.

14. Grantham JJ, Torres VE, Chapman AB, Guay-Woodford LM, Bae KT, King BF, $J$ r., et al. Volume progression in polycystic kidney disease. N Engl J Med. 2006;354:2122-30

15. Bae KT, Tao C, Zhu F, Bost JE, Chapman AB, Grantham JJ, et al. MRI-based kidney volume measurements in ADPKD: reliability and effect of gadolinium enhancement. Clin J Am Soc Nephrol. 2009:4:719-25.

16. Antiga L, Piccinelli M, Fasolini G, Ene-lordache B, Ondei P, Bruno S, et al. Computed tomography evaluation of autosomal dominant polycystic kidney disease progression: a progress report. Clin J Am Soc Nephrol. 2006; 1:754-60

17. Rule AD, Larson TS, Bergstralh EJ, Slezak JM, Jacobsen SJ, Cosio FG. Using serum creatinine to estimate glomerular filtration rate: accuracy in good health and in chronic kidney disease. Ann Intern Med. 2004;141:929-37.

18. Fontsere N, Salinas I, Bonal J, Bayes B, Riba J, Torres F, et al. Are prediction equations for glomerular filtration rate useful for the long-term monitoring of type 2 diabetic patients? Nephrol Dial Transplant. 2006;21:2152-8.

19. Chudleigh RA, Dunseath G, Evans W, Harvey JN, Evans P, Ollerton R, et al. How reliable is estimation of glomerular filtration rate at diagnosis of type 2 diabetes? Diabetes Care. 2007;30:300-5.

20. Agarwal R, Delanaye P. Glomerular filtration rate: when to measure and in which patients? Nephrol Dial Transplant. 2019:34:2001-7.

21. Delanaye P. Too much nephrology? The CKD epidemic is real and concerning. A CON view. Nephrol Dial Transplant. 2019:34:581-4.

22. Rule AD, Torres VE, Chapman AB, Grantham JJ, Guay-Woodford LM, Bae KT, et al. Comparison of methods for determining renal function decline in early autosomal dominant polycystic kidney disease: the consortium of radiologic imaging studies of polycystic kidney disease cohort. J Am Soc Nephrol. 2006;17:854-62.

23. Gaspari F, Perico N, Ruggenenti P, Mosconi L, Amuchastegui CS, Guerini E, et al. Plasma clearance of nonradioactive iohexol as a measure of glomerular filtration rate. J Am Soc Nephrol. 1995;6:257-63. 
24. Heath DA, Knapp MS, Walker WH. Comparison between inulin and 51Crlabelled edetic acid for the measurement of glomerular filtration-rate. Lancet. 1968;2:1110-2.

25. Price M. Comparison of creatinine clearance to inulin clearance in the determination of glomerular filtration rate. J Urol. 1972;107:339-40.

26. Ravine D, Gibson RN, Walker RG, Sheffield LJ, Kincaid-Smith P, Danks DM. Evaluation of ultrasonographic diagnostic criteria for autosomal dominant polycystic kidney disease 1. Lancet. 1994;343:824-7.

27. Phakdeekitcharoen B, Watnick TJ, Germino GG. Mutation analysis of the entire replicated portion of PKD1 using genomic DNA samples. J Am Soc Nephrol. 2001;12:955-63.

28. Brochner-Mortensen J. A simple method for the determination of glomerular filtration rate. Scand I Clin Lab Invest. 1972;30:271-4.

29. Levey AS, Stevens LA, Schmid CH, Zhang YL, Castro AF, 3rd, Feldman HI, et al. A new equation to estimate glomerular filtration rate. Ann Intern Med. 2009;150:604-12

30. Wibulpolprasert P, Tanomsingh N, Sungkarat W, et al. MRI-based kidney volume measurement in autosomal dominant polycystic kidney disease (ADPKD): Comparison of T2-weighted, ideal, and post-contrast T1-weighted sequences. J Med Assoc Thai. 2020;103(5):449-458.

31. Irazabal MV, Rangel LJ, Bergstralh EJ, Osborn SL, Harmon AJ, Sundsbak JL, et al. Imaging classification of autosomal dominant polycystic kidney disease: a simple model for selecting patients for clinical trials. J Am Soc Nephrol. 2015;26:160-72

32. Ruggenenti P, Gaspari F, Cannata A, Carrara F, Cella C, Ferrari S, et al. Measuring and estimating GFR and treatment effect in ADPKD patients: results and implications of a longitudinal cohort study. PLoS One. 2012; 7:e32533.

33. King BF, Reed JE, Bergstralh EJ, Sheedy PF, 2nd, Torres VE. Quantification and longitudinal trends of kidney, renal cyst, and renal parenchyma volumes in autosomal dominant polycystic kidney disease. J Am Soc Nephrol. 2000;11:1505-11.

34. Tokiwa S, Muto S, China T, Horie S. The relationship between renal volume and renal function in autosomal dominant polycystic kidney disease. Clin Exp Nephrol. 2011;15:539-45.

35. Grantham JJ. Clinical practice. Autosomal dominant polycystic kidney disease. N Engl J Med. 2008:359:1477-85.

36. Chapman AB, Guay-Woodford LM, Grantham JJ, Torres VE, Bae KT, Baumgarten DA, et al. Renal structure in early autosomal-dominant polycystic kidney disease (ADPKD): The Consortium for Radiologic Imaging Studies of Polycystic Kidney Disease (CRISP) cohort. Kidney Int. 2003;64: 1035-45.

37. Grantham JJ, Chapman AB, Torres VE. Volume progression in autosomal dominant polycystic kidney disease: the major factor determining clinical outcomes. Clin J Am Soc Nephrol. 2006;1:148-57.

\section{Publisher's Note}

Springer Nature remains neutral with regard to jurisdictional claims in published maps and institutional affiliations.

Ready to submit your research? Choose BMC and benefit from:

- fast, convenient online submission

- thorough peer review by experienced researchers in your field

- rapid publication on acceptance

- support for research data, including large and complex data types

- gold Open Access which fosters wider collaboration and increased citations

- maximum visibility for your research: over $100 \mathrm{M}$ website views per year

At $\mathrm{BMC}$, research is always in progress.

Learn more biomedcentral.com/submissions 